\title{
Association Between Platelet Count as a Noninvasive Marker and Ultrasonographic Grading in Patients With Nonalcoholic Fatty Liver Disease
}

\author{
Afagh Garjani ${ }^{1}$; Abdolrasoul Safaeiyan ${ }^{2}$; Manouchehr Khoshbaten ${ }^{3,}$ \\ ${ }^{1}$ Student Research Committee, Tabriz University of Medical Sciences, Tabriz, IR Iran \\ ${ }^{2}$ Department of Vital Statistics and Epidemiology, Faculty of Health and Nutrition, Tabriz University of Medical Sciences, Tabriz, IR Iran \\ ${ }^{3}$ Liver and Gastrointestinal Diseases Research Center, Emam Reza Educational Hospital, Tabriz, IR Iran \\ ${ }^{*}$ Corresponding Author: Manouchehr Khoshbaten, Liver and Gastrointestinal Diseases Research Center, Emam Reza Educational Hospital, Golgasht St., Tabriz, IR Iran. Tel: +98- \\ 4133347554, E-mail: mkhoshbaten@yahoo.com
}

Received: October 19, 2014; Revised: November 27, 2014; Accepted: December 27, 2014

\begin{abstract}
Background: Nonalcoholic fatty liver disease has become a worldwide challenge. Liver biopsy remains the single most reliable approach to determine the severity of this disease. As patients with nonalcoholic fatty liver disease require close follow-up, performing this invasive method repeatedly seems impractical; therefore, designing a noninvasive system to follow up patients has become a common interest. Objectives: We intended to investigate the association between platelet counts of patients with nonalcoholic fatty liver disease and the severity of their disease based on serum levels of liver enzymes and grade of fatty liver on ultrasonography.

Patients and Methods: One thousand, three hundred and five patients with nonalcoholic fatty liver disease were included in this descriptive study. Serum levels of aspartate aminotransferase (AST), alanine aminotransferase (ALT), alkaline phosphatase (ALP) and platelet counts of patients were measured. The grade of fatty liver was determined by abdominal ultrasonography.

Results: Of our study population, $54.3 \%(\mathrm{n}=708)$ were women. Patients with mild fatty liver on ultrasonography had lower platelet counts than those with moderate and severe fatty liver. However, no cutoff value of platelet count could reliably distinguish different grades of fatty liver. We found no significant association between platelet counts and serum levels of AST, ALT or ALP. However, we showed that male patients with abnormal levels of ALT had higher platelet counts.

Conclusions: Platelet count in nonalcoholic fatty liver disease can serve as a clue to the severity of disease, but it cannot be considered as a sole test to follow up patients.
\end{abstract}

Keywords:Non-alcoholic Fatty Liver Disease; Blood Platelets; Ultrasonography

\section{Background}

Nonalcoholic fatty liver disease (NAFLD) comprehends a spectrum of liver disorders ranging from simple fat accumulation in the hepatocytes (hepatic steatosis), to liver inflammation and hepatocyte injury (nonalcoholic steatohepatitis, NASH) with increasing levels of fibrosis to cirrhosis and hepatocellular carcinoma (HCC) in the worst-case scenario (1-3). Patients with NAFLD have a higher risk of morbidity and mortality (3-5). It is estimated to have a prevalence of $20 \%-30 \%$ in the western general population $(2,6,7)$. NAFLD has become the most common cause of chronic liver disease (8). In the Asian-Pacific region, the prevalence of NAFLD has been reported to range from $12 \%$ to $24 \%(3,7,8)$ and it is rapidly on the rise $(3,6)$. In Iran, the prevalence is $2.04 \%$ in the adult population (9) and 7.1\% in the adolescent population (10). In addition, NAFLD appears to have similar clinical and paraclinical features in Iran's population compared to other areas of the world (11).

For definite diagnosis and staging of NAFLD, liver biopsy remains the gold standard. Liver biopsy may, how- ever, over- or underestimates the stage of liver disease, because it only samples a very small portion of the liver. The invasive nature of this procedure plus practical difficulties and high expenses further justify the need for a safe and reliable approach for follow-up of patients with NAFLD (12-16). In the recent years, emergence of noninvasive methods has provided clinicians with the possibility to identify severe liver fibrosis. However, these approaches do not fully correlate with histological stage of NAFLD in the absence of substantial liver fibrosis $(16,17)$. In clinical practice, on the other hand, NAFLD is diagnosed based on ultrasonographic findings of fatty liver, elevated liver enzymes that cannot be otherwise explained and by excluding other causes of liver disease $(18,19)$.

Thrombopoietin (TPO) is a hormone produced mainly by hepatocytes, which promotes platelet production by the bone marrow (20). Studies indicated that the level of TPO is correlated to the degree of liver fibrosis and impairment of hepatocyte function (20, 21), both of which contribute to NAFLD (1-3). Therefore, peripheral platelet

Copyright ( ) 2015, Kowsar Corp. This is an open-access article distributed under the terms of the Creative Commons Attribution-NonCommercial 4.0 International License (http://creativecommons.org/licenses/by-nc/4.0/) which permits copy and redistribute the material just in noncommercial usages, provided the original work is properly cited. 
count can possibly reflect the severity of liver injury (22). A number of studies demonstrated an association between platelet counts and the severity of liver injury (2327), the results are controversial however. Several systems of scoring liver fibrosis such as the NAFLD fibrosis score (28), aspartate aminotransferase (AST)-to-platelet ratio index (APRI) (29) and the FIB-4 index (30) incorporated the platelet count as one of their variables emphasizing the potential role of platelet count as an indicator of liver injury. There is a definite need for further research regarding the association between platelet counts and noninvasive measures of the severity of NAFLD.

\section{Objectives}

In this study, we intended to investigate the association between platelet counts of patients with NAFLD and activity of their disease regarding clinical measures of the severity of NAFLD. To achieve this purpose, we specifically sought to assess platelet counts of patients in correspondence to their serum levels of liver enzymes and ultrasonographic grade of fatty liver.

\section{Patients and Methods}

\subsection{Study Design}

A total of 1305 patients with NAFLD were recruited from our outpatient clinic from December 2007 to April 2013 in this descriptive study.

The diagnosis of NAFLD was based on ultrasonographic findings of fatty liver, elevated serum levels of alanine aminotransferase (ALT) or aspartate aminotransferase (AST) that could not be otherwise explained, and by excluding other causes of liver disease. Other causes of chronic liver disease were eliminated if the patient met the following criteria: alcohol consumption less than 20 $\mathrm{g} / \mathrm{d}$ for men and less than $10 \mathrm{~g} / \mathrm{d}$ for women; no recent use of medications such as glucocorticoids, estrogens, tamoxifen, or amiodarone that may cause fatty liver; absence of other causes of liver disease indicated by normal results of laboratory tests including hepatitis B surface antigen (HBsAg), anti-hepatitis C antibody (anti-HCV), antinuclear antibodies (ANAs), anti-smooth muscle antibodies (ASMAs), Liver Kidney Microsomal type 1 antibody (anti-LKM1), gamma globulin levels, ceruloplasmin levels, ferritin levels and serum $\alpha-1$ antitrypsin levels (18).

Serum levels of liver enzymes including ALT, AST and alkaline phosphatase (ALP) and the platelet counts of patients were measured. Serum levels more than $40 \mathrm{IU} / \mathrm{L}$ for ALT and AST and more than $120 \mathrm{IU} / \mathrm{L}$ for ALP were considered abnormal. Patients with platelet counts less than 15 $\times 10^{4} / \mu \mathrm{L}$ were considered to have thrombocytopenia.

Abdominal ultrasonography was performed. Fatty liver was defined by at least two of three findings on abdominal ultrasonography; diffusely increased liver echogenicity greater than that of the kidneys or spleen, blurred intrahepatic vessels and attenuation of ultrasound pen- etration into the deep portions of the liver (31). In addition, the ultrasonographic grade of fatty liver defined as mild, moderate or severe was determined. Patients were assessed for the presence of an enlarged spleen.

The laboratory tests and abdominal ultrasonography in this study were performed before administration of therapy or within six months of therapy to avoid potential effects of treatment on their results.

\subsection{Statistical Analysis}

Statistical analysis was conducted using Statistical Package for the Social Sciences version 22 (IBM SPSS Statistics Chicago IL USA) and Minitab 16.2 statistical software(State College, Pennsylvania, United States). Pearson correlation was used to evaluate the association between platelet count and demographic, laboratory or ultrasonographic findings. t-test and one-way analysis of variance (ANOVA) were used for group comparisons. Categorical data were compared by chi-square and Cochran-Mantel-Haenszel tests. Sidak and Bonferroni tests were used for multiple comparisons. The normality of all continuous variables was tested using the Q-Q plot, and for variables without a normal distribution, appropriate mathematical transformation was applied to reform their deviation from normal. The diagnostic performance of the platelet count was assessed using the receiver operating characteristic (ROC) curve. Data were expressed as mean \pm standard error of mean (SEM) or as proportions (n). Error bars in the figures represent 95\% confidence interval (CI). Differences were considered statistically significant at $\mathrm{P}<0.05$.

\subsection{Ethical Concerns}

This study was approved by the ethics committee of Tabriz University of Medical Sciences. All the required information for this study were acquired as part of routine medical care for patients with NAFLD and no extra tests or costs were imposed on patients. The personal information of patients remained concealed throughout the study.

\section{Results}

One thousand, three hundred and five patients with NAFLD were included in the analysis. The characteristics of patients are summarized in Table 1.

The platelet counts in patients with NAFLD ranged from 2.3 to $49.4 \times 10^{4} / \mu \mathrm{L}$. Only $2.4 \%$ of patients $(\mathrm{n}=32)$ had thrombocytopenia, and none of them had an enlarged spleen on abdominal ultrasonography. The mean platelet count was significantly lower in males compared to females $\left(23.8 \pm 0.2 \times 10^{4} / \mu \mathrm{L} v\right.$ s. $\left.27.1 \pm 0.2 \times 10^{4} / \mu \mathrm{L}, \mathrm{P}=0\right)$. Of the study population, $48.2 \%$ were younger than 45 years $(n=629)$. There was no significant difference between the platelet counts of patients younger than 45 years and those 45 years old or older.

Platelet counts stratified by the ultrasonographic grade of fatty liver were as follows; mild: $25.3 \pm 0.2 \times 10^{4} / \mu \mathrm{L}$; mod- 
erate: $26 \pm 0.3 \times 10^{4} / \mu \mathrm{L}$; severe: $26.2 \pm 0.6 \times 10^{4} / \mu \mathrm{L}$. Patients with mild fatty liver had significantly $(P=0.042)$ lower platelet counts than those with moderate and severe fatty liver collectively $\left(25.3 \pm 0.2 \times 10^{4} / \mu \mathrm{L} v s .26 .1 \pm 0.3 \times 10^{4} / \mu \mathrm{L}\right)$. However, when we compared platelet counts within male and female patient groups, no significant difference was found between different grades of fatty liver in males. In females, on the other hand, platelet count was significantly lower in patients with mild fatty liver compared to those with moderate and severe fatty liver $(26.7 \pm 0.2 \times$ $10^{4} / \mu \mathrm{L} v$ s. $28 \pm 0.4 \times 10^{4} / \mu \mathrm{L}, \mathrm{P}=0.015$ ) (Figure 1 ). According to these findings, we executed a receiver operating characteristic (ROC) curve analysis to evaluate the diagnostic performance of platelet count to discriminate three grades of nonalcoholic fatty liver on ultrasonography. Remarkably, all ROC curves for detecting mild, moderate and severe fatty liver based on the platelet count were along the so-called line of no-discrimination. No optimal cutoff value for the platelet count could be determined to differentiate the three grades of fatty liver (Figures 2 , 3 and 4).

There was no significant difference between the platelet counts of patients with normal and abnormal AST or ALP. Regarding normal and abnormal ALT, the platelet count did not differ between the two groups in general; however, male subjects with abnormal ALT had higher ( $\mathrm{P}=$ $0.029)$ platelet counts than those with normal ALT (24.2 \pm $0.3 \times 10^{4} / \mu \mathrm{L} v$ s. $\left.23.1 \pm 0.3 \times 10^{4} / \mu \mathrm{L}\right)$. Of the study population, $50.6 \%$ of patients had platelet counts equal to or lesser than $25.2 \times 10^{4} / \mu \mathrm{L}(\mathrm{n}=661)$. When we compared serum levels of liver enzymes (i.e. ALT, AST and ALP) between the

\begin{tabular}{lc}
\hline \multicolumn{2}{|c}{ Table 1. Characteristics of NAFLD Patients Population ${ }^{\mathrm{a}, \mathrm{b}}$} \\
\hline Demographics & \multicolumn{1}{c}{ Data } \\
\hline Age, $\mathrm{y}$ & $46.4 \pm 0.3$ \\
Gender, male/female & $597(45.7) / 708(54.3)$
\end{tabular}

\section{Hematology}

Platelet count, $\times 10^{4} / \mu \mathrm{L} \quad 25.6 \pm 0.1$

\section{Hepatology panel}

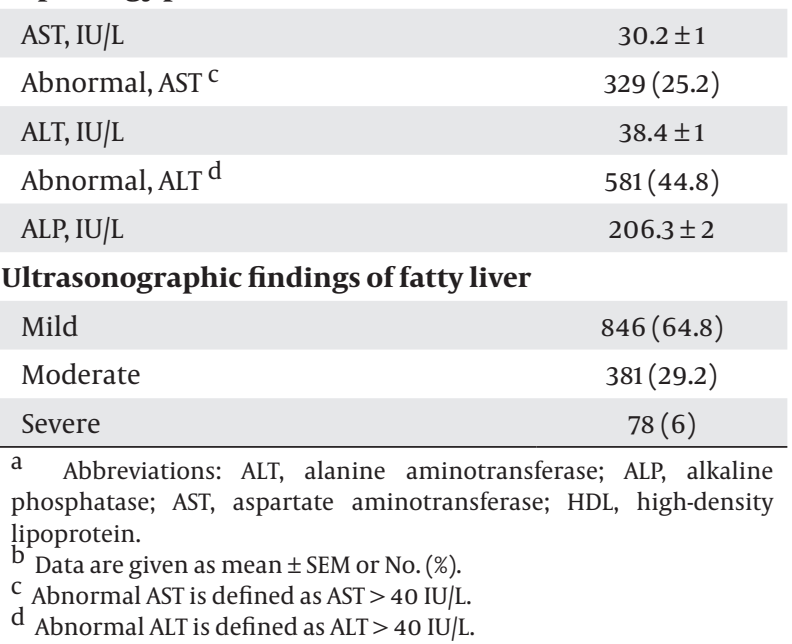

two groups, the only significant difference was found in male patients whose natural logarithm of the serum levels of ALT was higher $(\mathrm{P}=0.018)$ with platelet counts $>$ $25.2 \times 10^{4} / \mu \mathrm{L}$ than with values $\leq 25.2 \times 10^{4} / \mu \mathrm{L}(52.7 \pm 1[95 \%$ CI 48.9- 56.8] IU/L vs. $47 \pm 1$ [95\% CI 44.3- 49.8] IU/L).

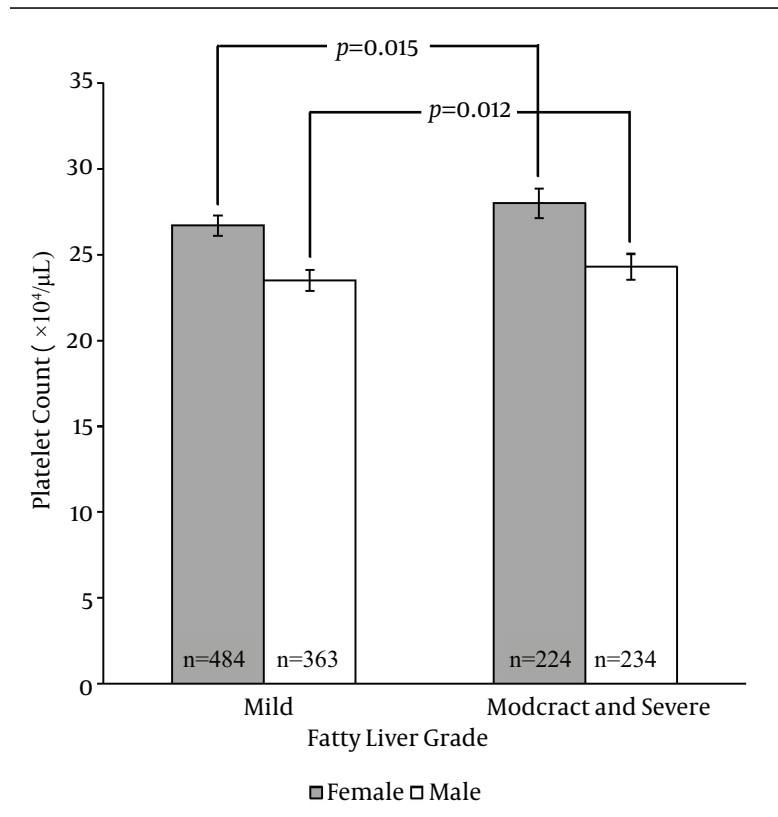

Figure 1. Platelet Counts of Female and Male Patients With Mild Fatty Liver Compared to Those With Moderate and Severe Fatty Liver

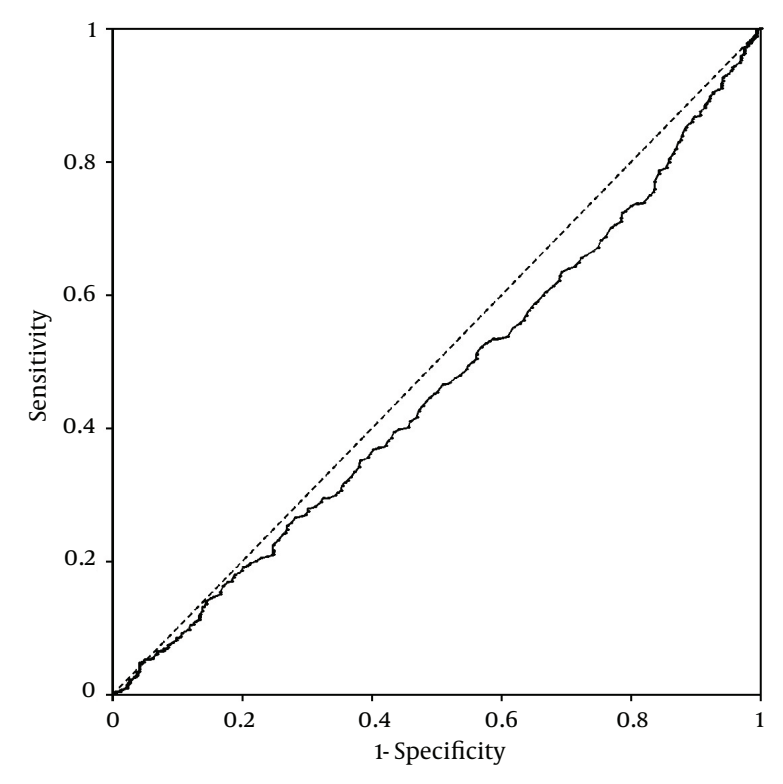

Figure 2. Receiver Operating Characteristic (ROC) Curve for Detecting Mild Nonalcoholic Fatty Liver by Ultrasonography Based on the Platelet Count 


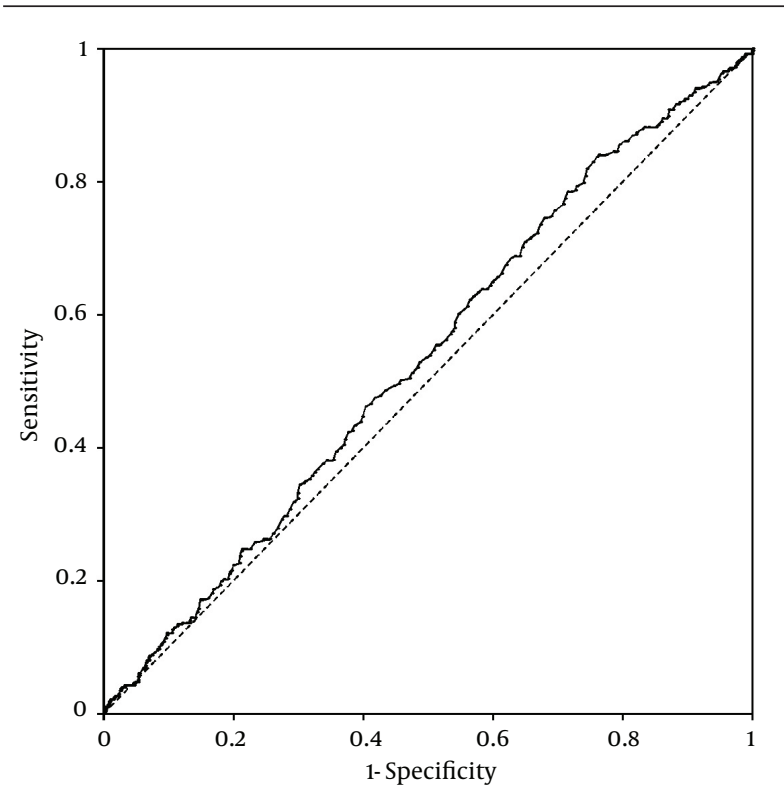

Figure 3. Receiver Operating Characteristic (ROC) Curve for Detecting Moderate Nonalcoholic Fatty Liver by Ultrasonography Based on the Platelet Count

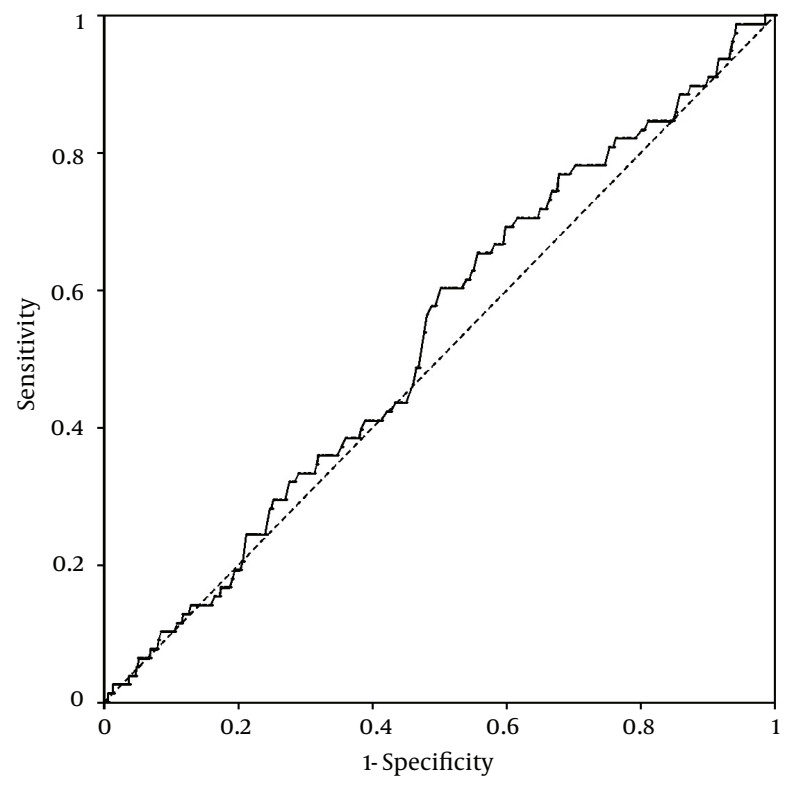

Figure 4. Receiver Operating Characteristic (ROC) Curve for Detecting Severe Nonalcoholic Fatty Liver by Ultrasonography Based on the Platelet Count

\section{Discussion}

The present study was planned to determine the association between platelet counts of patients with NAFLD and certain features of their disease. In this study, diagnosis of NAFLD was made based on clinical, laboratory and ultrasonographic findings, and no liver biopsy was performed (12-16).

As mentioned earlier, few studies in this field yielded controversial results. A number of prior investigations demonstrated a negative correlation between platelet count and severity of NAFLD for liver fibrosis. The most current study in this respect was a major cohort study conducted by Yoneda et al. (23), in which a low platelet count was presented as a measure of severe liver fibrosis. The fact that platelet count can merely predict severe stages of liver fibrosis and its insignificant role to differentiate milder stages of fatty liver is common in all these studies (23-25). A possible explanation for this is splenic sequestration of platelets, which might occur in patients with severe liver fibrosis and cirrhosis (32). It is also probable that liver injury causes reduced platelet production in the bone marrow due to defective TPO release $(20,21$, 32 ). There are, on the other hand, recent studies which have cast doubt on the association between liver injury and the levels of TPO $(33,34)$. However, this subject is beyond the scope of our discussion.

Contrary to these findings, the results of our study indicated that patients with moderate and severe nonalcoholic fatty liver on ultrasonography have higher platelet counts than those with mild fatty liver. This finding is consistent with those of some recent researches $(26,35$, 36). Cao et al. for instance, demonstrated that patients with NASH have higher platelet counts than those with hepatic steatosis (26). This rather contradictory result may be due to the fact that platelet counts increase in response to inflammation (37), and hepatic inflammation is the channel through which hepatic steatosis leads to liver injury and fibrosis (1-3). Only $2.4 \%$ of patients with NAFLD had thrombocytopenia, and none of them had an enlarged spleen on ultrasonography. It is because not many of our patients had severe liver fibrosis or cirrhosis and that steatohepatitis contributed to the pathogenesis of our NAFLD patients with moderate and severe fatty liver and higher platelet counts. However, in the absence of liver biopsy, these data must be interpreted with caution. Moreover, we concluded that no cutoff value for platelet count could reliably predict the grade of fatty liver on abdominal ultrasonography.

Surprisingly, in our study, the difference between the platelet counts of patients with different grades of fatty liver was significant only in women. This observation may signify the influence of gender on NAFLD. The literature on this issue is insufficient. Studies have shown that NASH is more prevalent in women (38). It may be the case that these variations between men and women are due to the failure of abdominal ultrasonography to differentiate severe forms of hepatic steatosis and NASH. Bearing in mind that inflammation may raise the platelet count, it is possible that male patients with moderate and severe fatty liver had hepatic steatosis, while their female counterparts had steatohepatitis. Further investigation is necessary to address these questions.

Regarding the liver enzymes, ALT and AST, the results 
of our study did not show any significant difference between the platelet counts of patients with normal and those with abnormal liver enzymes in general. It can be assumed that elevated liver enzymes do not indicate hepatic inflammation and injury. This observation accords with some prior studies which failed to show any correlation between serum levels of liver enzymes and the severity of liver injury (39). However, this finding seems not to apply to men in our study, who had higher platelet counts with abnormal serum levels of ALT. Its reason is not clear, but it is possible that serum levels of ALT are superior to abdominal ultrasonography in detecting steatohepatitis in men. Taken together, it is suggested to perform further researches regarding the role of gender in NAFLD.

Taking into account the necessity to implement noninvasive methods for follow-up of patients with NAFLD, this study attempted to determine whether platelet count of NAFLD patients could predict the activity of their disease. This study suggests that platelet count could be used as a clue to the severity of NAFLD, but it cannot be considered as a sole noninvasive test to follow up patients.

\subsection{Study Limitation}

There were some limitations in this study. The severity of NAFLD was defined mostly based on ultrasonographic findings, while liver biopsy remains the single most reliable method to determine the severity of NAFLD. However, performing liver biopsy on such a large number of patients seems both impractical and unethical. Furthermore, ultrasonography cannot differentiate fat infiltration from hepatic fibrosis, also is not always reliable in obese patients (40). Another limitation was that our study population included only NAFLD patients, while evaluating NAFLD patients against a control group might have yielded more dependable results. Finally, future cohort studies on this topic are recommended to further clarify the association between platelet count and noninvasive measures of NAFLD.

\section{Authors' Contributions}

Study concept and design: Khoshbaten and Garjani. Acquisition of data: Khoshbaten and Garjani. Analysis and interpretation of data: Safaeiyan and Garjani. Drafting of the manuscript: Garjani. Critical revision of the manuscript for important intellectual content: Khoshbaten, Safaeiyan, and Garjani. Statistical analysis: Safaeiyan and Garjani. Study supervision: Khoshbaten.

\section{References}

1. Dowman JK, Tomlinson JW, Newsome PN. Systematic review: the diagnosis and staging of non-alcoholic fatty liver disease and non-alcoholic steatohepatitis. Aliment Pharmacol Ther. 2011;33(5):525-40.

2. de Alwis NM, Day CP. Non-alcoholic fatty liver disease: the mist gradually clears. J Hepatol. 2008;48 Suppl 1:S104-12.

3. Chitturi S, Farrell GC, Hashimoto E, Saibara T, Lau GK, Sollano JD, et al. Non-alcoholic fatty liver disease in the Asia-Pacific region: definitions and overview of proposed guidelines. J Gastroenterol Hepatol. 2007;22(6):778-87.

4. Adams LA, Lymp JF, St Sauver J, Sanderson SO, Lindor KD, Feldstein $A$, et al. The natural history of nonalcoholic fatty liver disease: a population-based cohort study. Gastroenterology. 2005;129(1):113-21.

5. Treeprasertsuk S, Lopez-Jimenez F, Lindor KD. Nonalcoholic fatty liver disease and the coronary artery disease. Dig Dis Sci. 2011;56(1):35-45.

6. Clark JM. The epidemiology of nonalcoholic fatty liver disease in adults. JClin Gastroenterol. 2006;40 Suppl 1:S5-10.

7. Vernon G, Baranova A, Younossi ZM. Systematic review: the epidemiology and natural history of non-alcoholic fatty liver disease and non-alcoholic steatohepatitis in adults. Aliment Pharmacol Ther. 2011;34(3):274-85.

8. Farrell GC, Larter CZ. Nonalcoholic fatty liver disease: from steatosis to cirrhosis. Hepatology. 2006;43(2 Suppl 1):S99-S112.

9. Jamali R, Khonsari M, Merat S, Khoshnia M, Jafari E, Bahram Kalhori A, et al. Persistent a lanine aminotransferase elevation among the general Iranian population: prevalence and causes. World $J$ Gastroenterol. 2008;14(18):2867-71.

10. Alavian SM, Mohammad-Alizadeh AH, Esna-Ashari F, Ardalan G, Hajarizadeh B. Non-alcoholic fatty liver disease prevalence among school-aged children and adolescents in Iran and its association with biochemical and anthropometric measures. Liver Int. 2009;29(2):159-63.

11. Khoushbaten M, Fatahi E, Soymi MH, Tarzamani MK, Farhang S, Majidi G, et al. Clinico-biochemical comparison of patients with nonalcoholic fatty liver disease and healthy population. Zahedan J Research Med Sci. 2009;11(1):13-21.

12. Vuppalanchi R, Chalasani N. Nonalcoholic fatty liver disease and nonalcoholic steatohepatitis: Selected practical issues in their evaluation and management. Hepatology. 2009;49(1):306-17.

13. Younossi ZM. Review article: current management of non-alcoholic fatty liver disease and non-alcoholic steatohepatitis. Aliment Pharmacol Ther. 2008;28(1):2-12.

14. Erickson SK. Nonalcoholic fatty liver disease. J Lipid Res. 2009;50 Suppl:S412-6.

15. Guha IN, Parkes J, Roderick PR, Harris S, Rosenberg WM. Noninvasive markers associated with liver fibrosis in non-alcoholic fatty liver disease. Gut. 2006;55(11):1650-60.

16. Martinez SM, Crespo G, Navasa M, Forns X. Noninvasive assessment of liver fibrosis. Hepatology. 2011;53(1):325-35.

17. McPherson S, Stewart SF, Henderson E, Burt AD, Day CP. Simple non-invasive fibrosis scoring systems can reliably exclude advanced fibrosis in patients with non-alcoholic fatty liver disease. Gut. 2010;59(9):1265-9.

18. Farrell GC, Chitturi S, Lau GK, Sollano JD, Asia-Pacific Working Party on N. Guidelines for the assessment and management of non-alcoholic fatty liver disease in the Asia-Pacific region: executive summary. J Gastroenterol Hepatol. 2007;22(6):775-7.

19. Schwenger KJ, Allard JP. Clinical approaches to non-alcoholic fatty liver disease. World J Gastroenterol. 2014;20(7):1712-23.

20. Weksler BB. Review article: the pathophysiology of thrombocytopenia in hepatitis $C$ virus infection and chronic liver disease. Aliment Pharmacol Ther. 2007;26 Suppl 1:13-9.

21. Giannini E, Borro P, Botta F, Fumagalli A, Malfatti F, Podesta E, et al. Serum thrombopoietin levels are linked to liver function in untreated patients with hepatitis $\mathrm{C}$ virus-related chronic hepatitis. J Hepatol. 2002;37(5):572-7.

22. Karasu Z, Tekin F, Ersoz G, Gunsar F, Batur Y, Ilter T, et al. Liver fibrosis is associated with decreased peripheral platelet count in patients with chronic hepatitis B and C. Dig Dis Sci. 2007;52(6):1535-9.

23. Yoneda M, Fujii H, Sumida Y, Hyogo H, Itoh Y, Ono M, et al. Platelet count for predicting fibrosis in nonalcoholic fatty liver disease. $J$ Gastroenterol. 2011;46(11):1300-6.

24. Neuschwander-Tetri BA, Clark JM, Bass NM, Van Natta ML, UnalpArida A, Tonascia J, et al. Clinical, laboratory and histological associations in adults with nonalcoholic fatty liver disease. Нераtology. 2010;52(3):913-24.

25. Park KS, Lee YS, Park HW, Seo SH, Jang BG, Hwang JY, et al. Factors associated or related to with pathological severity of nonalco- 
holic fatty liver disease. Korean J Intern Med. 2004;19(1):19-26.

26. Cao W, Zhao C, Shen C, Wang Y. Cytokeratin 18, alanine aminotransferase, platelets and triglycerides predict the presence of nonalcoholic steatohepatitis. PLoS One. 2013;8(12).

27. Syn WK, Nightingale P, Bateman JM. Nonalcoholic fatty liver disease in a district general hospital: clinical presentation and risk factors. Hepatol Int. 2008;2(2):190-5.

28. Angulo P, Hui JM, Marchesini G, Bugianesi E, George J, Farrell GC, et al. The NAFLD fibrosis score: a noninvasive system that identifies liver fibrosis in patients with NAFLD. Hepatology. 2007;45(4):846-54

29. Wai CT, Greenson JK, Fontana RJ, Kalbfleisch JD, Marrero JA, Conjeevaram HS, et al. A simple noninvasive index can predict both significant fibrosis and cirrhosis in patients with chronic hepatitis C. Hepatology. 2003;38(2):518-26.

30. Sterling RK, Lissen E, Clumeck N, Sola R, Correa MC, Montaner J, et al. Development of a simple noninvasive index to predict significant fibrosis in patients with HIV/HCV coinfection. Hepatology. 2006;43(6):1317-25.

31. Hamaguchi M, Kojima T, Itoh Y, Harano Y, Fujii K, Nakajima T, et al. The severity of ultrasonographic findings in nonalcoholic fatty liver disease reflects the metabolic syndrome and visceral fat accumulation. Am J Gastroenterol. 2007;102(12):2708-15.

32. Afdhal N, McHutchison J, Brown R, Jacobson I, Manns M, Poordad
F, et al. Thrombocytopenia associated with chronic liver disease. J Hepatol. 2008;48(6):1000-7.

33. Temel T, Cansu DU, Temel HE, Ozakyol AH. Serum thrombopoietin levels and its relationship with thrombocytopenia in patients with cirrhosis. Hepat Mon. 2014;14(5).

34. Yilmaz B, Basar O, Altinbas A, Ekiz F, Aktas B, Ozturk G, et al. The utility of thrombopoietin in predicting liver fibrosis in chronic hepatitis B. Int J Clin Exp Med. 2014;7(5):1430-4.

35. Sung KC, Kim BS, Cho YK, Park DI, Woo S, Kim S, et al. Predicting incident fatty liver using simple cardio-metabolic risk factors at baseline. BMC Gastroenterol. 2012;12:84.

36. Johansson HE, Haenni A, Zethelius B. Platelet counts and liver enzymes after bariatric surgery. J Obes. 2013;2013:567984.

37. Esmon CT. The impact of the inflammatory response on coagulation. Thromb Res. 2004;114(5-6):321-7.

38. Pan JJ, Fallon MB. Gender and racial differences in nonalcoholic fatty liver disease. World J Hepatol. 2014;6(5):274-83.

39. Khosravi S, Alavian SM, Zare A, Daryani NE, Fereshtehnejad SM, Daryani NE, et al. Non-alcoholic fatty liver disease and correlation of serum alanin aminotransferase level with histopathologic findings. Hepat Mon. 2011;11(6):452-8.

40. Razavizade M, Jamali R, Arj A, Talari H. Serum parameters predict the severity of ultrasonographic findings in non-alcoholic fatty liver disease. Hepatobiliary Pancreat Dis Int. 2012;11(5):513-20. 